\title{
Development of a unique anti-AML immune therapy consisting of cord blood HSCT and cord blood stem cell-derived dendritic cell (CB-DC) vaccination
}

\author{
Colin de Haar*, Maud Plantinga, Nina Blokland, Lotte Spel, Marianne Boes, Stefan Nierkens, Jaap Jan Boelens \\ From Society for Immunotherapy of Cancer 28th Annual Meeting \\ National Harbor, MD, USA. 8-10 November 2013
}

\section{Background}

Development of novel (immune) therapies is of utmost importance to improve survival in relapsed pediatric-AML (acute myeloid leukemia). We aim to develop a powerful and safe therapy consisting of 2 synergistic components: Cord Blood (CB) HSCT and vaccination with CB-derived Wilms Tumor-1 (WT1) mRNA-electroporated dendritic cells (DCs).

\section{Materials \& methods}

After isolation, the CD34+ CB stem cells were cultured using a two-step protocol. First, they were expanded using a combination of (growth) factors (Flt3L, SCF, IL-3 and IL-6). Next, the cells were differentiated towards DCs for one week using medium containing Flt3L, SCF, GM-CSF, IL- 4 and human serum followed by a CYTOMIX (IL-1 $\beta$, IL-6, TNF- $\alpha$ and PGE2)-induced maturation for the last 24 hours. Finally, the CB-DC culture was electroporated with WT1-mRNA and their phenotype (cell surface markers) and function (migration and antigen presentation) were assessed.

\section{Results}

Using the two-step protocol a total cell expansion of 300-500 fold was achieved. Based on surface marker expression, at least 5 different DC subsets could be distinguished in our CB-DC cultures. Since no differences in antigen presentation capacity between the DC subsets were detected, the whole CB-DC culture was used in all phenotypic and functional assays. The maturation using
CYTOMIX induced upregulation of costimulatory molecules and CCR7. These cells were also functional, showing enhanced CCR7-dependent migration towards CCL21 in a trans-well migration assay. Finally, the stimulation of WT1-specific T cells by the CB-DCs, matured using CYTOMIX and electroporated with WT1 mRNA, confirmed presentation of WT1 antigens.

\section{Conclusion}

We have developed and tested an in vitro system for culturing large amounts of DCs from the CD34+ CB stem cells. Both the phenotypic and functional data support the use of the whole CB-DC culture as vaccine. The next step will be to translate the preclinical protocol to GMP production of a clinical grade vaccine.

Published: 7 November 2013

\section{doi:10.1186/2051-1426-1-S1-P206}

Cite this article as: de Haar et al:: Development of a unique anti-AML immune therapy consisting of cord blood HSCT and cord blood stem cell-derived dendritic cell (CB-DC) vaccination. Journal for ImmunoTherapy of Cancer 2013 1(Suppl 1):P206. 